\title{
CULTIVOS DE COBERTURA EN AMBIENTE SUBHÚMEDO BONAERENSE: POÁCEAS Y LEGUMINOSAS ANTECESORES DE GIRASOL
}

\author{
Sá Pereira, e. DE ${ }^{1}$; Arroquy, G. ${ }^{1} ;$ Minoldo, G. ${ }^{2} ;$ \\ Galantini, J. ${ }^{3 ;}$ Holzmann, A. ${ }^{4} \&$ Ducos, I. ${ }^{4}$
}

\begin{abstract}
RESUMEN
Los cultivos de cobertura (CC) se utilizan tradicionalmente para controlar problemas de erosión, mejorar la captación, distribución y almacenaje de agua, reducir la compactación, mejorar los balances de carbono y nitrógeno del suelo. Este trabajo pretendió aportar resultados zonales en cuanto a las contribuciones de CC sobre eficiencia de uso de los recursos y productividad del girasol. Durante 2017 y 2018 se ensayaron en Coronel Suárez (Sudoeste de Buenos Aires) seis CC como antecesores de girasol. Se evaluaron: agua en el suelo, materia seca, nitrógeno y rendimiento. La diferencia en el contenido de agua edáfica al secado de los CC respecto al barbecho convencional (BC) varió entre -8 y $15 \mathrm{~mm}$. La eficiencia de uso de agua (EUA) con respecto al testigo, oscilaron entre 5,59 $\mathrm{kg} \mathrm{MS} \mathrm{mm}^{-1}$ en vicia $\left(\mathrm{V}_{1}\right)$ y de $16,16 \mathrm{~kg} \mathrm{MS} \mathrm{mm}^{-1}$ en avena (A). Los mayores rendimientos de girasol se obtuvieron con $\mathrm{V}_{1}\left(2379 \mathrm{~kg} \mathrm{ha}^{-1}\right)$ y los menores con $\mathrm{A}\left(1561 \mathrm{~kg} \mathrm{ha}^{-1}\right)$.
\end{abstract}

Key words: vicia, centeno, materia seca, costo hídrico, producción.

\begin{abstract}
Cover crops: poace and legumes as antecessors of the cultivation of sunflower in subhumedo bonaerense environment.

Cover crops (CC) are used traditionally to control erosion problems, improve water collection, distribution and storage, reduce compaction, and improve soil carbon and nitrogen balances. This work aimed to provide zonal results regarding the contributions of $\mathrm{CC}$ on resource use efficiency and sunflower productivity. During 2017 and 2018, six CCs as sunflower predecessors in Coronel
\end{abstract}

1.- AER INTA Coronel Suárez (EEA C. Naredo). Sauce Corto 589. (7540) Coronel Suárez. Buenos Aires, Argentina. Email: desapereira.eduardo@inta.gob.ar

2.- Departamento de Agronomía, Universidad Nacional del Sur. San Andrés 800. (8000) Bahía Blanca, Argentina 3.- Comisión de investigaciones Científicas (CIC, Pcia. Bs. As.) - CERZOS-CIC. San Andrés 800. (8000) Bahía Blanca, Argentina.

4.- Criadero de semillas "El Cencerro". H. Yrigoyen 824. (7540) Coronel Suárez. Buenos Aires, Argentina. Manuscrito recibido el 22 de mayo de 2020 y aceptado para su publicación el 11 de septiembre de 2020.

Sá Pereira E. de, Arroquy G., Minoldo G., Galantini J., Holzmann A. \& Ducos I. Cultivos de cobertura en ambiente subhúmedo bonaerense: poáceas y leguminosas antecesores de girasol (Hellianthus annus L.) FAVE - Ciencias Agrarias 19 (2): 7-21. CC BY-NC-SA 4.0 
Suárez (Southwest of Buenos Aires) were tested. Soil water and dry matter, nitrogen and vegetable yield were evaluated. The difference in edaphic water content upon drying of the $\mathrm{CC}$ with respect to the conventional fallow (BC) varied between -8 and $15 \mathrm{~mm}$. The water use efficiency (EUA) ranged from $5.59 \mathrm{~kg} \mathrm{MS} \mathrm{mm}^{-1}$ in vetch $\left(\mathrm{V}_{1}\right)$ and $16.16 \mathrm{~kg} \mathrm{MS} \mathrm{mm}^{-1}$ in oats $(\mathrm{A})$. The highest yields were obtained with $\mathrm{V}_{1}\left(2379 \mathrm{~kg} \mathrm{ha}^{-1}\right)$ and the lowest with $\mathrm{A}\left(1561 \mathrm{~kg} \mathrm{ha}^{-1}\right)$.

Key words: vetch, rye, dry matter, water cost, production.

\section{INTRODUCCIÓN}

En los sistemas productivos de la región central del sudoeste (RCS) de la provincia de Buenos Aires (Argentina), principalmente en la zona de Coronel Suárez, actualmente se observa una gran difusión de la agricultura continua bajo siembra directa (SD). Existe un predominio de soja (Glycine $\max (\mathrm{L}$.) Merr.), en monocultivo, en doble cultivo, en secuencias con trigo (Triticum aestivum L.) o cebada (Hordeum vulgare) y en menor medida en rotación con maíz (Zea maíz L.) y girasol (Hellianthus annus L.). Los cultivos de verano se cosechan entre marzo y mayo, los cultivos de invierno como el trigo y la cebada entre diciembre y enero. Las siembras de los próximos cultivos de verano se llevan a cabo entre los meses de octubre y noviembre por lo cual, dependiendo si el antecesor es un cultivo de verano o invierno, el período de barbecho puede extenderse de 5 a 9 meses. Durante un período tan largo es altamente probable la ocurrencia de pérdida de agua del suelo, aún bajo sistemas conservacionistas (Basanta et al., 2008). Una alternativa interesante de manejo bajo este tipo de sistemas, sería la inclusión de cultivos de cobertura (CC) con el objeto de mejorar la eficiencia de utilización del agua (EUA) entre cultivos y lograr otros beneficios derivados de las funciones que cumplen los $\mathrm{CC}$ tales como: la cobertura y protección del suelo, la captura de nutrientes como nitrógeno (N) y fósforo (P) y su posterior liberación resultado de su descomposición, el aporte de carbono al suelo, el control de malezas, plagas y enfermedades, y la depresión del nivel freático (Sá Pereira, 2013).

En la región pampeana húmeda los $\mathrm{CC}$ de invierno se establecen en el período de tiempo entre la cosecha del cultivo de verano y la siembra del siguiente cultivo de verano. Durante ese período, estos CC no son pastoreados, incorporados, ni cosechados (Álvarez y Scianca, 2007). En el sudoeste bonaerense, también se pueden establecer $\mathrm{CC}$ entre un cultivo de invierno (trigo o cebada) y el próximo cultivo de verano (soja, maíz o girasol). Esta sucesión de cultivos es comúnmente realizada por los productores de la RCS, principalmente en planteos de agricultura permanente. Allí, en los últimos años, el área sembrada con girasol se ha visto particularmente disminuida (RIAN, 2018). En la región, no existe información del comportamiento de este cultivo como sucesor de un CC de invierno. En este planteo los CC, podrían favorecer también la recuperación de los niveles de cobertura del suelo derivados de los escasos residuos de cosecha aportados por el girasol. Además, $\mathrm{CC}$ con leguminosas contribuyen con el 
aporte de nutrientes, principalmente nitrógeno proveniente de la fijación biológica de $\mathrm{N}(\mathrm{FBN})$ y de la descomposición de residuos (Sá Pereira, 2013). El uso de vicia (Vicia sativa $\mathrm{L}$ ) como CC en maíz en suelos de textura franco-arenosa del centro oeste de Buenos Aires, tendría la potencialidad de ser adoptada, con la finalidad de reducir la dosis de fertilizantes nitrogenados (Rillo et al., 2013). Los CC pueden producir biomasa, lo que disminuye el riesgo de erosión. Además, pueden secuestrar $\mathrm{N}$ con potencialidad de ser lixiviado, para su posterior liberación al cultivo de verano siguiente (Thorup-Kristensen et al., 2003).

En función de los antecedentes presentados, el objetivo de este trabajo fue evaluar la viabilidad y las contribuciones de diferentes $\mathrm{CC}$, eficiencia de uso de agua y sus efectos sobre los distintos parámetros de producción del girasol.

\section{MATERIALES Y MÉTODOS}

El ensayo se condujo en el campo experimental perteneciente al Criadero "El Cencerro", Coronel Suárez, provincia de Buenos Aires (37²7'56"S,6151'53'W; $227 \mathrm{~m} \mathrm{snm}$ ) durante la campaña 2017/18 sobre un suelo clasificado como Argiudol Típico (Soil Survey Staff, 2014); serie Laprida 8 - capacidad de uso (IIs)/índice de productividad 73,53, con al menos veinte años previos bajo agricultura continua. Se evaluaron siete tratamientos: Vicia villosa INTA: $30 \mathrm{~kg} \mathrm{ha}^{-1}\left(\mathrm{~V}_{1}\right)$, Vicia villosa Guasch: $30 \mathrm{~kg} \mathrm{ha}^{-1}$ (V2), Secale cereale (Centeno Don Ewald: $105 \mathrm{~kg} \mathrm{ha}^{-1}$ ) (C), Avena sativa (Avena Marita INTA: $140 \mathrm{Kg} \mathrm{ha}^{-1}$ ) (A), Trifolum pratense L. (Trébol Rojo Redomón: $18 \mathrm{~kg} \mathrm{ha}^{-1}$ ) (T), Avena sativa Marita
INTA: $121 \mathrm{~kg} \mathrm{ha}^{-1}+$ Vicia villosa INTA: 26 $\mathrm{kg} \mathrm{ha}^{-1}\left(\mathrm{~A}+\mathrm{V}_{1}\right)$, como especies y asociaciones de $\mathrm{CC}$ y un barbecho convencional sin $\mathrm{CC}$ como testigo (BC). El diseño fue en bloques completamente aleatorizados con cuatro (4) repeticiones, en subparcelas de 12 surcos de $10 \mathrm{~m}$ de largo (superficie: 1,95 $\mathrm{m} \times 10 \mathrm{~m}=19,5 \mathrm{~m} 2$ ). Las borduras se sembraron con Vicia villosa INTA.

El control de malezas en BC se realizó en forma mecánica (con azada), en el resto de las parcelas no se controlaron las malezas. La siembra de los CC se realizó el 15/03/17, con sembradora experimental para labranza convencional junto a una aplicación de fosfato diamónico $\left(60 \mathrm{~kg} \mathrm{ha}^{-1}\right)$ en toda la parcela de ensayo. Las semillas de leguminosas fueron inoculadas específicamente. Las parcelas con centeno y avena se fertilizaron en el mes de julio con $40 \mathrm{~kg} \mathrm{~N} \mathrm{ha}^{-1}$ en forma de urea al voleo. En agosto, en estas parcelas se realizó el despunte de las plantas (corte del tercio superior), con el fin de favorecer el rebrote. Para el secado de los CC, el $17 / 10 / 17$ se aplicaron $2,5 \mathrm{~L} \mathrm{ha}^{-1}$ de glifosato $48 \%+500 \mathrm{~cm}^{3} \mathrm{ha}^{-1}$ de $2,4 \mathrm{D}$ éster.

En tres momentos de corte $(22 / 08 / 17$, $18 / 09 / 17$ y 12/10/17) de cada CC se tomaron muestras de biomasa aérea de una superficie de $0,25 \mathrm{~m}^{2}$, se determinó el peso del material verde. Luego las muestras se secaron en estufa $\left(60^{\circ} \mathrm{C}\right)$ para la determinación de producción de materia seca total aérea (MS).

El cultivo de girasol se sembró el $15 / 11 / 17$, con una densidad de 55000 semillas ha-1 (52 cm entre hileras). En preemergencia, se aplicó fluorcloridona $\left(1,5 \mathrm{~L} \mathrm{ha}^{-1}\right)$ + acetoclor $\left(1,5 \mathrm{~L} \mathrm{ha}^{-1}\right)+$ lambdacialotrina $25 \%\left(25 \mathrm{~cm}^{3} \mathrm{ha}^{-1}\right)$. El 03/01/18 se evaluó el índice de aspecto/desarrollo (IAD) de las plantas de girasol en escala de 1 a 5 donde: $1=$ malo, $2=$ regular, 3=bueno, 4=muy bue- 
no y $5=$ excelente. Conjuntamente se midió la superficie de área foliar $\left(\mathrm{SAF}, \mathrm{cm}^{2}\right)$, efectuándose 30 observaciones por tratamiento y repetición. En antesis (25/01/18), se tomaron muestras de plantas y se evaluó la producción de MS. A cosecha (29/03/18) se evaluaron el número total de plantas ha-1 y plantas quebradas $\mathrm{ha}^{-1} \mathrm{y}$ se determinó el rendimiento $\left(\mathrm{kg} \mathrm{ha}^{-1}\right)$.

Al momento de siembra, secado de los CC y siembra del girasol se tomaron muestras de suelo de las capas de 0-20 y 20-50 $\mathrm{cm}$ de profundidad. Se determinó el contenido de $\mathrm{N}$ de nitratos $\left(\mathrm{N}-\mathrm{NO}^{3}\right)$ del suelo (Mulvaney, 1996), la humedad gravimétrica $\left(\% \mathrm{H}^{\circ}\right)$, el uso consuntivo (UC), costo hídrico $(\mathrm{CH})$ y se calculó la eficiencia de uso del agua (EUA) de los CC.

Para calcular el AET se utilizó la fórmula: $\operatorname{AET}(\mathrm{mm})=\Sigma\left(\% \mathrm{H}^{\circ}\right.$ gravimétrica $\mathrm{x}$ DAP x Prof.) $/ 10$

Donde $\% \mathrm{H}^{\circ}$ gravimétrica $=$ porcentaje de humedad gravimétrica. DAP $=$ densidad aparente del suelo $\left(\mathrm{g} \mathrm{cm}^{3}\right)$, Prof. $=$ profundidad de muestreo $(\mathrm{cm})$.

El CH se calculó utilizando la fórmula: $\mathrm{CH}(\mathrm{mm})=\mathrm{AET} \mathrm{CC}-\mathrm{AET} \mathrm{BC}$

Donde AET $\mathrm{CC}=\mathrm{AET}$ del $\mathrm{CC}$ al momento del secado $(\mathrm{mm})$; AET $\mathrm{BC}=\mathrm{AET}$ del $\mathrm{BC}$ al momento del secado ( $\mathrm{mm})$.

El UC se calculó con la fórmula propuesta por López \& Arrué, (1997):

$\mathrm{UC}(\mathrm{mm})=$ AET inicial $(\mathrm{mm})+\mathrm{PP}$ (mm) - AET final (mm)

Donde AET Inicial= AET Inicial se considera al momento de la siembra del CC $(\mathrm{mm})$. AET Final $=$ AET final se considera al secado del CC (mm); PP= PP ocurridas entre la siembra y el secado del CC.
La EUA en se calculó con la fórmula:

EUA $\left(\mathrm{kg} \mathrm{ha}^{-1} \mathrm{~mm}^{-1}\right)=\mathrm{BA} / \mathrm{UC}$

Donde $\mathrm{BA}=$ biomasa aérea $\left(\mathrm{kg} \mathrm{ha}^{-1}\right)$; $\mathrm{UC}=\mathrm{UC}$ del cultivo.

Durante el período de estudio, se registraron las precipitaciones diarias con estación meteorológica móvil a $500 \mathrm{~m}$ de distancia del ensayo y valores de evapotranspiración diarios (ETP) del Servicio Meteorológico Nacional a 2000 m en línea recta (SMN, 2018).

Para el análisis estadístico, se utilizó el software INFOSTAT (Di Rienzo et al., 2011). Se realizaron análisis de la varianza (ANAVA) y test de diferencias medias significativas (DMS) de Fisher.

\section{RESULTADOS Y DISCUSION}

Las precipitaciones y la ETP diaria (mm) ocurridas durante el ciclo agrícola 2017-18 en el área de estudio se muestran la Figura 1. En ella se distinguieron cuatro periodos cuya duración fue definida por algunas fases del ciclo de desarrollo de los cultivos: siembra de los CC de invierno (SCC), interrupción del crecimiento de los $\mathrm{CC}$ con herbicida (SeCC), siembra del girasol (SG), antesis del girasol (FG) y cosecha de cultivo de renta (CchG).

Las precipitaciones históricas acumuladas en el área de Coronel Suárez (SMN) para los periodos entre fases anteriores promedian: $443 \mathrm{~mm}$ entre marzo y octubre, $82 \mathrm{~mm}$ en noviembre, $165 \mathrm{~mm}$ entre diciembre y enero y $179 \mathrm{~mm}$ entre febrero y marzo. Cabe destacar que las precipitaciones ocurridas el año 2017 alcanzaron los $922 \mathrm{~mm}$, superando en un 17\% al promedio histórico para Coronel Suárez de 
790 mm (SMN, 2018) y que, ese año, las condiciones de humedad para el crecimiento de los CC y acumulación de agua durante el barbecho convencional fueron próximas a capacidad de campo. Contrariamente, respecto al periodo entre siembra y cosecha de girasol, las precipitaciones alcanzaron tan solo el $55 \%$ de la media histórica para esos meses, resultando en condiciones hídricas muy adversas durante el crecimiento y período crítico del cultivo de girasol, que en general, no afectaron el rendimiento promedio de la zona.
Producción de materia seca de los CC, consumo y eficiencia de uso del agua

La producción de MS de los CC varió entre 2956 y 8549 kg MS ha-1, con valores superiores en los tratamientos de gramíneas puras y que incluyeron gramíneas y leguminosas $(p=0,001)$ (Figura 2, Foto 1a y b). Estas diferencias en la cantidad de biomasa producida se manifestaron con mayor intensidad en el segundo y tercer corte de los diferentes CC. Hasta el primer periodo de crecimiento evaluado, fin de macollaje de A/

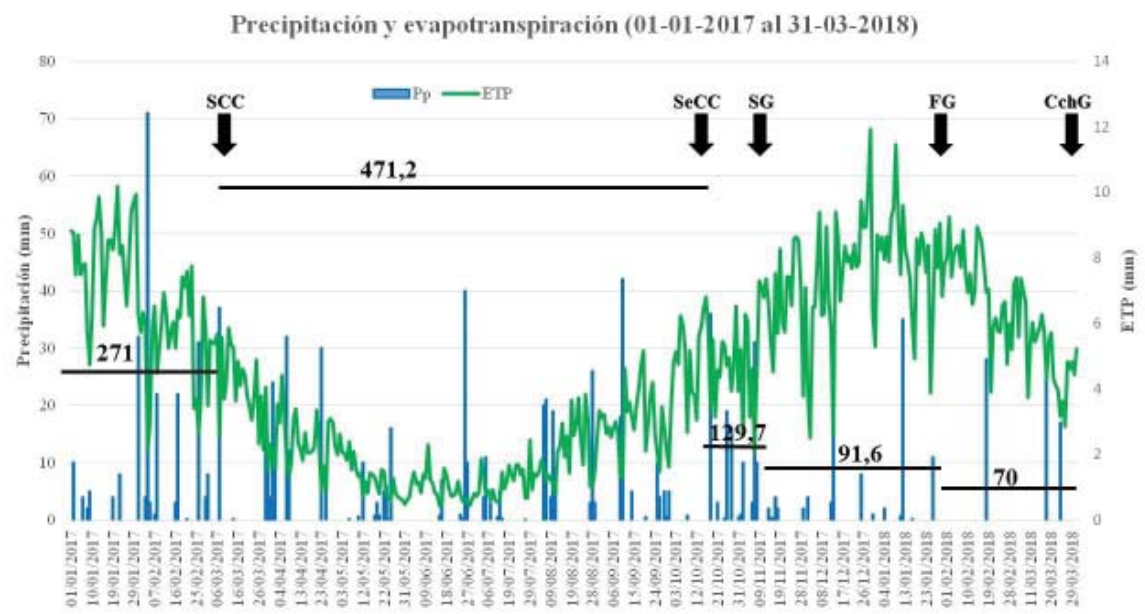

Figura 1: Precipitaciones decádicas (barras verticales) para el ciclo agrícola 2017 (15 de marzo) a 2018 (31 de marzo) ocurridas en el Criadero “El Cencerro”. Las líneas horizontales indican las precipitaciones acumuladas en cuatro periodos del ciclo: 15 de marzo al 17 de octubre, 18 de octubre al 13 de noviembre, 14 de noviembre al 24 de enero y 25 de enero al 30 de marzo. La curva continua de evolución representa la evapotranspiración potencial decádica (ETP) datos SMN (Aerostación Coronel Suárez). Siembra de cultivos de cobertura (SCC), secado de los cultivos de cobertura (SeCC), siembra de girasol (SG), floración de girasol (FG) y cosecha de girasol (CchG).

Figure 1: Decadal precipitation (vertical bars) for the agricultural cycle from 2017 (March 15) to 2018 (March 31) occurred at the "El Cencerro" Farm. The horizontal lines indicate accumulated rainfall in four periods of the cycle: March 15 to October 17, October 18 to November 13, November 14 to January 24, and January 25 to March 30. The continuous evolution curve represents the decadal potential evapotranspiration (ETP) SMN data (Coronel Suárez Aerostation). Sowing of cover crops (SCC), drying of cover crops (SeCC), sowing of sunflower $(S G)$, flowering of sunflower $(F G)$ and harvest of sunflower (CchG).

Revista FAVE - Ciencias Agrarias 19 (2) 2020 
principio encañazón de $C$, en general se detectaron menores rendimientos de MS de las leguminosas puras vs las gramíneas solas o consociadas; $\left(\mathrm{V}_{1}=\mathrm{V}_{2}=\mathrm{T}<\mathrm{C}=\mathrm{A}=\mathrm{A}+\mathrm{V}_{1}\right)$.

Ambos cultivares de vicia presentaron las menores producciones de MS durante todo el ciclo $(p=0,001)$. El trébol rojo, a principios de primavera mostró niveles de producción de MS superiores a la vicia pura $(p=0,001)$, lo que se mantuvo hasta la interrupción de los CC. La mayor producción de MS de A y $\mathrm{C}$, sugiere un importante secuestro de nutrientes, principalmente $\mathrm{N}$, potencialmente susceptible a ser lixiviado en el suelo $\left(\mathrm{V}_{1}=\mathrm{V}_{2}<\mathrm{T}<\mathrm{A}+\mathrm{V} 1=\mathrm{C}<\mathrm{A}\right)$.
El cultivar Avena Marita, reconocido como medianamente susceptible a roya de la hoja, mostró un comportamiento regular. Las condiciones de humedad y temperatura en 2017, fueron favorables para el desarrollo de esta enfermedad en estado vegetativo, con un otoño cálido, invierno poco riguroso y primaveras cálidas, aunque también promovieron un rápido crecimiento y rebrote, principalmente después del despunte, en el mes de agosto. Este comportamiento determinó que fuera la gramínea de mayor $(\mathrm{p}=0,001)$ producción de MS evaluada, principalmente en la segunda y tercera época de muestreo, seguida del C como CC.

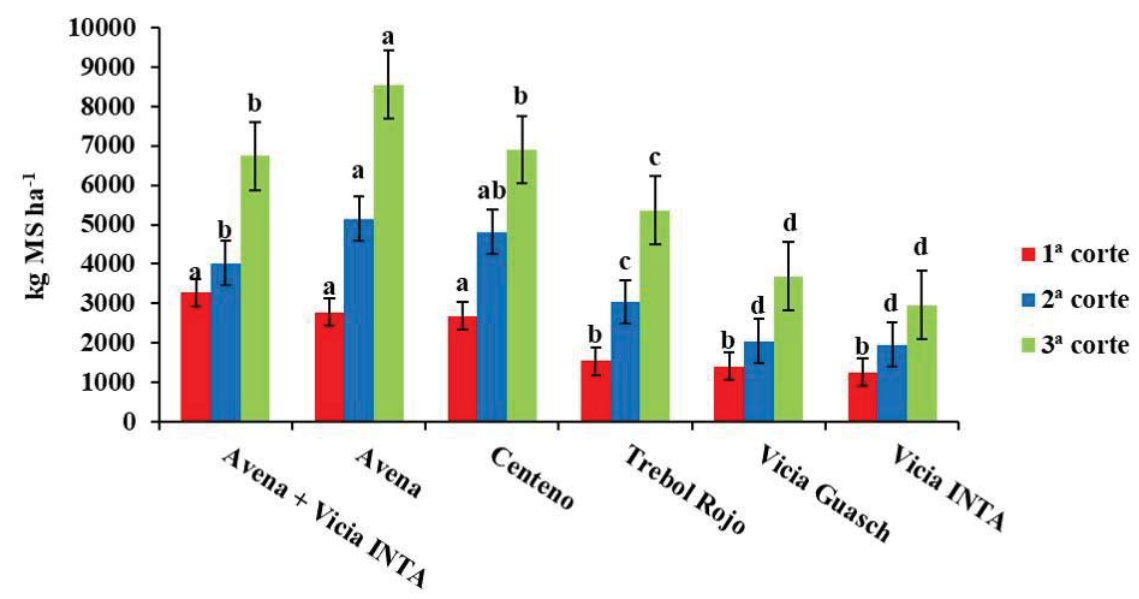

Antecesores Cultivos de cobertura

Figura 2: Producción de materia seca total aérea (MS) de los CC, 2017 en cada momento de corte. Letras diferentes indican diferencias mínimas significativas (Fisher, $\alpha \leq 0,05$ ) entre CC. Líneas verticales sobre cada columna indican el error estándar de la media.

Figure 2: Production of total aerial dry matter (DM) of the CC in each cutting season: 2017. For each CC growth period, different letters indicate minimal significant differences (Fisher, $\alpha \leq$ 0.05). Vertical line above each column indicates the standard error of the mean. 
Foto 1: a) Cultivos de cobertura (CC) de trébol rojo con su máximo crecimiento en octubre de 2017 b) CC Vicia villosa al centro, avena a la izquierda y avena + vicia a la derecha y c) Post-antesis de girasol en enero de 2018. Criadero "El Cencerro" Coronel Suárez.

Photo 1: a) Cover crops of Red Clover with its maximum growth in October 2017 b) CC of vetch in the center accompanied by oats and oats + vetch to the left and right respectively and c) Post-anthesis of sunflower in January 2018. "El Cencerro” Hatchery Coronel Suárez.

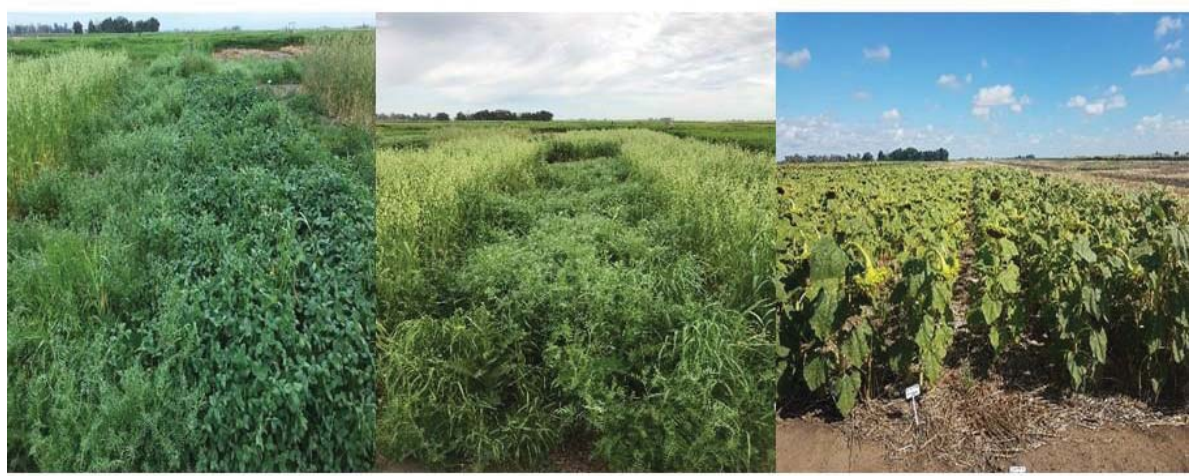

El contenido de agua total en el suelo y el uso consuntivo de los CC al momento del secado fue similar en todos los casos (Tabla 1). Las diferencias en el contenido de agua respecto al $\mathrm{BC}$ fueron positivas para $\mathrm{V}_{1} \mathrm{~V}_{2}$ y C. Estos CC no sólo no afectaron la dotación hídrica del suelo al momento de la siembra, sino que el cultivo siguiente contó con unos 5 a $15 \mathrm{~mm}$ extra respecto del BC. $\mathrm{Al}$ contrario, con la $\mathrm{A}, \mathrm{T}, \mathrm{A}+\mathrm{V}_{1}$, estas diferencias fueron negativas y se determinó un costo hídrico para el cultivo de girasol de entre 1 y $8 \mathrm{~mm}$. La eficiencia de uso del agua (EUA) oscilaron entre un mínimo de $5,8 \mathrm{~kg} \mathrm{MS} \mathrm{mm}^{-1}$ con $\mathrm{V}_{1}$ y un máximo de $16,8 \mathrm{~kg} \mathrm{MS} \mathrm{mm}^{1}$ con A. Avena siempre expresó valores altos de EUA. Estas son coincidentes con lo reportado por Sá Pereira (2013). En la RCS con precipitaciones promedio de $790 \mathrm{~mm}$ se podría esperar que en la mayoría de los años los CC no afecten a la disponibilidad de agua en el suelo al momento de la siembra de girasol. Estos resultados son similares a lo reportado por Sá Pereira et al. (2016) quien evaluó en las campañas 2012 y 2013 costos hídricos del orden de 10 a $20 \mathrm{~mm}$.

Los diferentes CC antecesores impactaron en forma diferencial sobre la producción de MS del girasol $(p=0,0479)$. En antesis los rendimientos fueron $3961\left(\mathrm{~V}_{1}\right)$ $\geq 3505\left(\mathrm{~V}_{2}\right) \geq 2994(\mathrm{BC}) \geq 2576(\mathrm{~A}+\mathrm{V})=$ 


\section{E. de Sá Pereira et al.}

Tabla 1: Contenido de agua total al momento de secado de los CC(Atscc) en mm hasta los $50 \mathrm{~cm}$, uso consuntivo de los CC (UCcc) (mm), materia seca (MS kg/ha-1), eficiencia de uso del agua de los CC (EUAcc) (kg MS mm-1 agua), diferencia en el contenido de agua del suelo para los tratamientos de avena (A), trébol (T), avena + vicia (A+V1), vicia (V1), vicia (V2), centeno (C) y testigo barbecho convencional al momento de secado de los CC, para el año 2017 en (\% y mm). Test de Fisher $(p<0,05)$.

Table 1: Total water content at the time of drying of CC (Atscc) in mm up to $50 \mathrm{~cm}$, consumptive use of CC (UCcc) ( $\mathrm{mm}$ ), dry matter (DM kg / ha-1), efficiency of water use of the CC (EUAcc) ( $\mathrm{kg}$ MS mm-1 water), difference in the soil water content for the treatments of oats (A), clover $(T)$, oats + common vetch $(A+V 1)$, common vetch (V1), common vetch (V2), rye (C) and conventional fallow control at the time of drying of the CC, for the year 2017 in (\% and mm). Fisher's test $(p<0.05)$.

\begin{tabular}{|c|c|c|c|c|c|c|}
\hline \multirow{3}{*}{ Tratamiento } & \multirow{3}{*}{ Atscc (mm) } & \multirow{3}{*}{$\operatorname{Uccc}(\mathrm{mm})$} & \multirow{3}{*}{$\begin{array}{c}\text { MS } 3^{\text {er }} \text { corte } \\
\left(\mathrm{kg} \mathrm{ha}^{-1}\right)\end{array}$} & \multirow{3}{*}{$\begin{array}{l}\text { EUAcc }(\mathrm{kg} \\
\left.\text { MS mm }{ }^{-1}\right)\end{array}$} & \multicolumn{2}{|c|}{ Diferencia de agua con BC } \\
\hline & & & & & \multicolumn{2}{|c|}{ secado CC } \\
\hline & & & & & $\mathrm{mm}$ & $\%$ \\
\hline$A$ & 131,65 & 514,94 & $8549 a$ & $16,60 \mathrm{a}$ & $-8,08$ & $-6,14$ \\
\hline$T$ & 136,06 & 510,53 & $5358 \mathrm{c}$ & $10,49 \mathrm{c}$ & $-3,67$ & $-2,70$ \\
\hline $\mathrm{A}+\mathrm{V}_{1}$ & 138,52 & 508,07 & $6734 \mathrm{~b}$ & $13,25 \mathrm{~b}$ & $-1,21$ & $-0,87$ \\
\hline $\mathrm{BC}$ & 139,73 & 506,86 & & & & \\
\hline$V_{1}$ & 144,35 & 502,24 & $3690 \mathrm{~d}$ & $7,34 \mathrm{~d}$ & 4,62 & 3,20 \\
\hline $\mathrm{V}_{2}$ & 145,23 & 501,36 & $2956 \mathrm{~d}$ & $5,89 \mathrm{~d}$ & 5,5 & 3,79 \\
\hline $\mathrm{C}$ & 154,63 & 491,56 & $6901 \mathrm{~b}$ & $14,03 \mathrm{~b}$ & 14,9 & 9,64 \\
\hline $\mathrm{p}$ & ns $(p=0,51)$ & ns $(p=0,51)$ & $p<0,0001$ & $p<0,0001$ & & \\
\hline
\end{tabular}

$2451(\mathrm{~T})=2446(\mathrm{C}) \geq 2278(\mathrm{~A}), \mathrm{kg} \mathrm{MS} \mathrm{ha}^{-1}$ (Figura 3). A partir de estos resultados se evidenció que la cantidad de MS total en floración de girasol manifestó una tendencia inversamente proporcional respecto a los niveles crecientes de producción de MS de los diferentes CC. Similares resultados, obtuvieron Ranells y Wagger, (1996), Trinsoutrot et al. (2000) y Sá Pereira et al. (2017). Dichos autores, relacionaron la mayor concentración de lignina y relación $\mathrm{C}: \mathrm{N}$ determinada en los residuos de las gramíneas respecto a las leguminosas, con una más lenta descomposición y consiguiente liberación de nutrientes (Aulakh et al., 1991 y Mary et al., 1996), disponibles para el cultivo siguiente. Estas desiguales velocidades de transformación de los residuos entre leguminosas y gramíneas, habrían impactado directamente en la producción de MS de girasol (Foto 1c). Sá Pereira et al. (2017a), aplicando modelos matemáticos de simulación para la descomposición de materia orgánica $(\mathrm{MO})$ de diferentes $\mathrm{CC}$ visualizaron estas diferencias. La producción de MS de girasol tuvo valores extremos superiores luego de la $\mathrm{V}_{1}$, intermedios con $\mathrm{V}_{2}, \mathrm{BC}$, $\mathrm{A}+\mathrm{V}, \mathrm{T}$ y $\mathrm{C}$ e inferiores luego de la $\mathrm{A}(\mathrm{p}=$ 0,0479, Figura 3). 


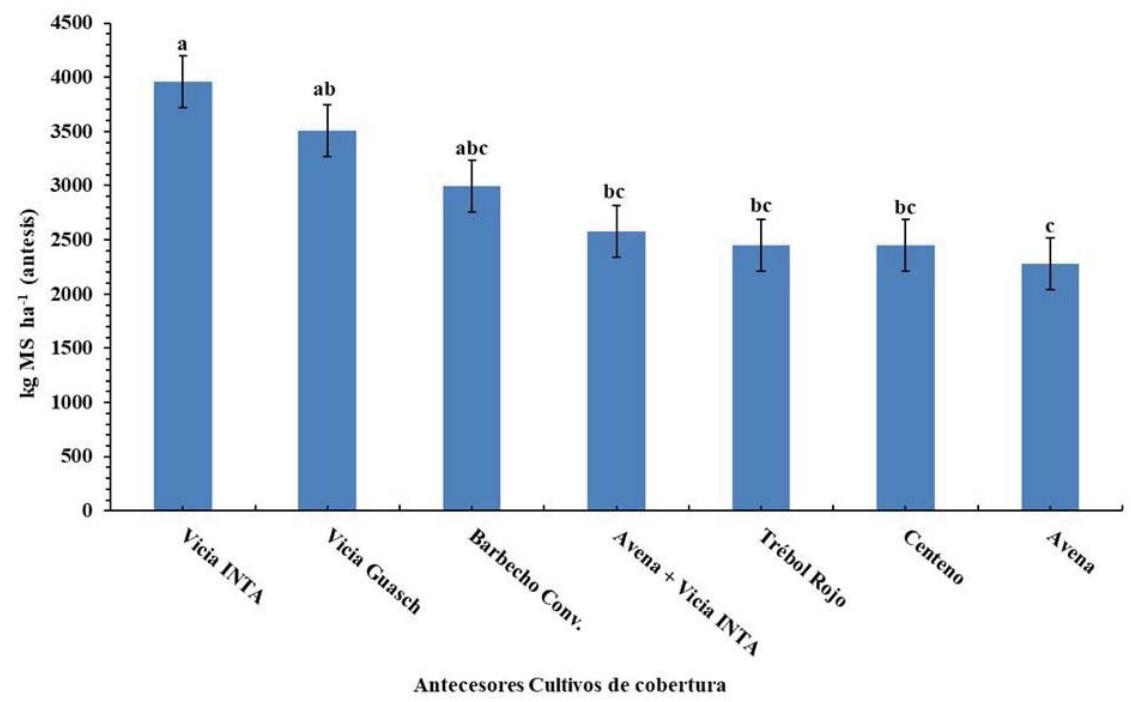

Figura 3: Producción de materia seca total aérea (MS; $\mathrm{kg}$ ha-1) de girasol en antesis en función de los CC antecesores. Letras diferentes indican diferencias minimas significativas (Fisher, $\alpha \leq$ 0,05). Lineas verticales sobre cada columna indican el error estándar de la media.

Figure 3: Production of total aerial dry matter (DM; $\mathrm{kg} \mathrm{ha-1)}$ of sunflower in anthesis with the different CC ancestors. Different letters indicate minimal significant differences (Fisher, $\alpha \leq 0.05$ ). Vertical line above each column indicates the standard error of the mean.

Las mayores SAF del girasol en antesis, se obtuvieron con $\mathrm{BC}$ y $\mathrm{CC}$ leguminosas, puras o consociadas, sin diferencias entre ellos $(\mathrm{p}=0,0001)$, (Figura 4). La menor $\mathrm{SAF}$ con $\mathrm{C}$ y A como antecesores podría deberse a la inmovilización de $\mathrm{N}$ asociada al volumen de residuos con alta relación $\mathrm{C}: \mathrm{N}$ aportados en el suelo. Por otro lado, la duración del barbecho entre el secado de los CC y la siembra del girasol fue de tan solo 20 días, lo que demoró la descomposición.

La mayor SAF de las plantas de girasol con antecesor $\mathrm{T}, \mathrm{BC}, \mathrm{A}+\mathrm{V}_{1} \mathrm{~V}_{1}$ y $\mathrm{V}_{2}$ indicarían un efecto del $\mathrm{N}$ residual en el suelo, proveniente de la FBN y la descomposición de residuos de $\mathrm{V}_{1}, \mathrm{~V}_{2}$ y $\mathrm{T}$ desde su secado.
Resultados similares, reportó Sá Pereira (2013) sobre Vicia villosa como CC antecesor de maíz en la RCS, si bien, los niveles de $\mathrm{N}$ en el suelo a la siembra del girasol fueron superiores en el tratamiento $\mathrm{BC}$ y menores con vicia (Figura 6). El antecesor vicia pondría en disponibilidad del cultivo, $\mathrm{N}$ residual más tempranamente hacia el periodo previo a botón floral del girasol, algo similar a lo que ocurrió con maíz en ensayos conducidos con los mismos CC en la RCS llevadas adelante por Sá Pereira et al. (2014).

El número total de plantas de girasol, no se vio influenciado significativamente por los diferentes CC $(\mathrm{p}=0,36)$, si bien pudo detectarse cierta tendencia destacable de la población obtenida luego de V1, (Figura 5). 
E. de Sá Pereira et al.

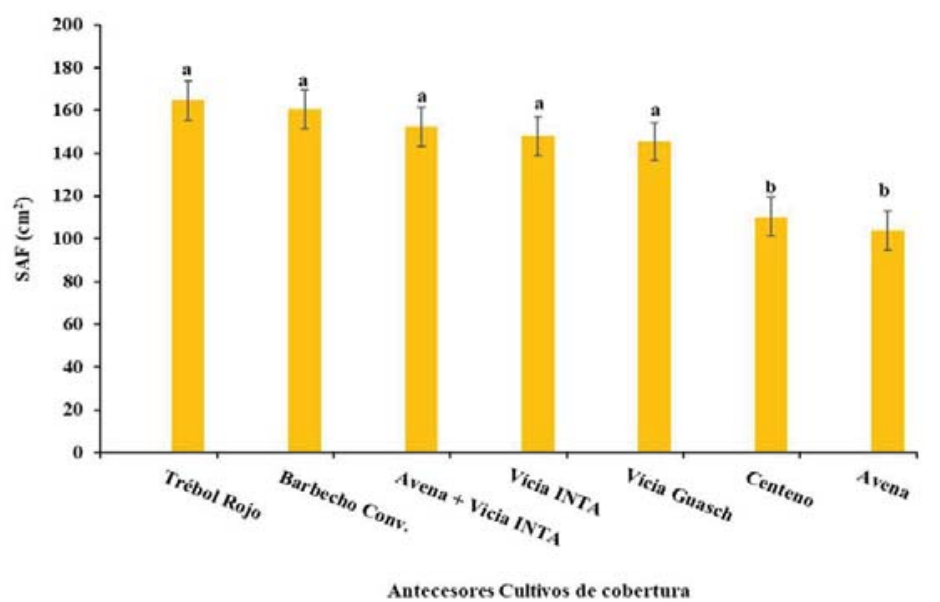

Figura 4: Superficie foliar (SAF) en $\mathrm{cm}^{2}$ del cultivo de girasol en antesis con los diferentes CC como antecesores. Letras diferentes indican diferencias mínimas significativas (Fisher, $\alpha \leq 0,05$ ). Lineas verticales sobre cada columna indican el error estándar de la media.

Figure 4: Leaf area (SAF) in cm 2 of the sunflower culture in anthesis with the different CC as ancestors. Different letters indicate minimal significant differences (Fisher, $\alpha \leq 0.05$ ). Vertical line above each column indicates the standard error of the mean.

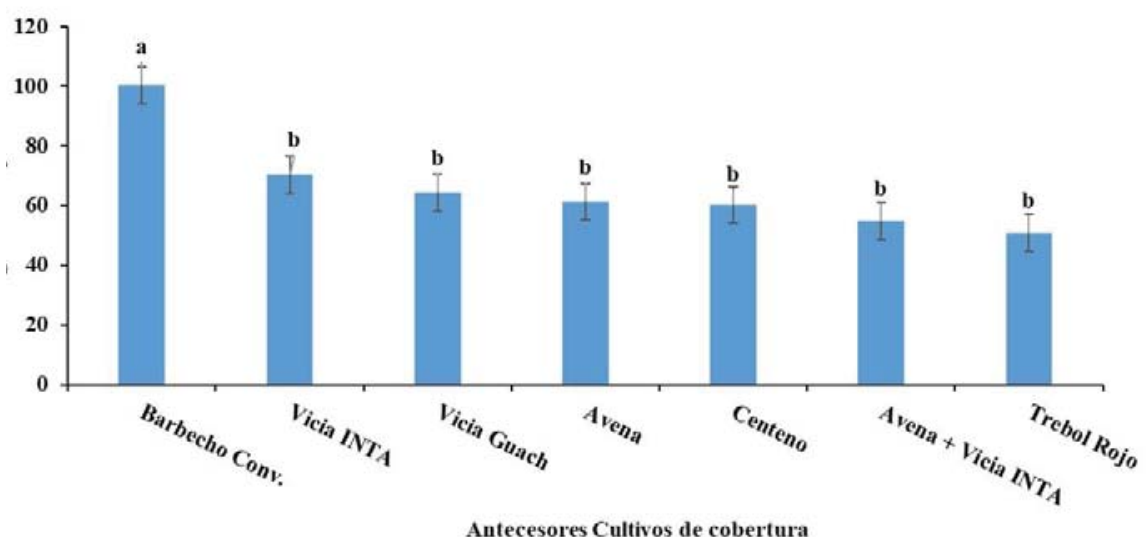

Figura 5: Número de plantas de girasol (Pl. ha-1) según CC antecesores. Letras diferentes indican diferencias minimas significativas (Fisher, $\alpha \leq 0,05$ ). Lineas verticales sobre cada columna indican el error estándar de la media.

Figure 5: Number of sunflower plants (Pl. ha-1) according to CC ancestors. Different letters indicate minimal significant differences (Fisher, $\alpha \leq 0.05$ ). Vertical line above each column indicates the standard error of the mean. 
El contenido de N-nitratos (N-NO3-) disponible en los primeros $50 \mathrm{~cm}$ de profundidad del suelo a la siembra del girasol, fue superior en el tratamiento BC (100,3 kg N ha-1) respecto a los CC: $70,3\left(\mathrm{~V}_{1}\right)=$ $64,4\left(\mathrm{~V}_{2}\right)=61,3(\mathrm{~A})=60,3(\mathrm{C})=54,9(\mathrm{~A}+\mathrm{V})$ $=50,9 \mathrm{~kg} \mathrm{~N}^{-1}(\mathrm{~T}),(\mathrm{p}=0,0179$ ), (Figura 6). Este mayor nivel de N-NO3- en el suelo con BC, muy posiblemente se debió al sistema de labranza utilizado, con remoción de suelo. Esto puso a disposición del cultivo de renta, una mayor cantidad de $\mathrm{N}$ inicial, respecto a los CC.

La Figura 7 muestra los IAD en las plantas de girasol en prefloración. Plantas con IAD excelente resultaron aquellas con antecesor $\mathrm{V}_{1}, \mathrm{~V}_{2}$ y BC $(\mathrm{p}=0,0080)$, intermedios en $\mathrm{A}$ y $\mathrm{C}$ y los más bajos correspondieron a los tratamientos $\mathrm{T}$ y $\mathrm{A}+\mathrm{V}_{1}$, probablemente debido al momento de secado muy tardío de los CC, con alto contenidos de tejido lignificado, con escasas o nulas precipitaciones desde la emergencia, que hicieron, en los primeros días de enero no se manifestasen todavía, los efectos benéficos de aportes de $\mathrm{N}$ por parte del $\mathrm{T}$ y la $\mathrm{V}_{1}$ en asociación con A previo a la siembra de girasol. Este índice mostró una disminución relativa con la incorporación de gramíneas solas o asociadas y trébol solo, aumentando con vicia y $\mathrm{BC}$ como antecesores. El momento dentro del ciclo fenológico en el cual se suspende el crecimiento mediante herbicidas sistémicos (secado) determina cuanta biomasa se genera $\mathrm{y}$, por ende, cuanto $\mathrm{N}$ es retenido en la misma (Clark et al., 1995), citado por Vanzolini et al. (2010). Por este motivo, y con el fin de acumular mayor cantidad de $\mathrm{N}$

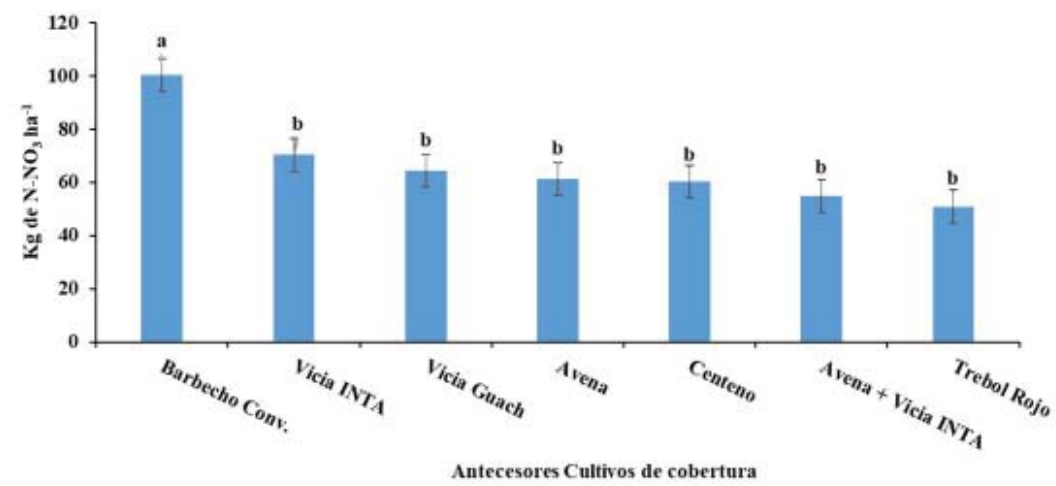

Figura 6: Nitrógeno disponible de 0-50 cm del suelo ( $\mathrm{kg} \mathrm{ha-1)} \mathrm{al} \mathrm{momento} \mathrm{de} \mathrm{la} \mathrm{siembra} \mathrm{del}$ cultivo de girasol luego de diferentes cultivos de cobertura antecesores. Letras distintas indican diferencias significativas $(\alpha \leq 0,05)$. Las lineas verticales en cada columna muestran el error estándar de la media.

Figure 6: Soil available Nitrogen in 0-50 cm depth ( $\mathrm{kg} \mathrm{ha-1),} \mathrm{at} \mathrm{the} \mathrm{time} \mathrm{of} \mathrm{planting} \mathrm{the} \mathrm{sunflower}$ crop with different CC predecessor. Different letters indicate minimal significant differences (Fisher, $\alpha \leq 0.05)$. Vertical line above each column indicates the standard error of the mean. 
fijado, fechas tardías de secado serían más convenientes para suplantar la fertilización nitrogenada del cultivo de maíz (Rillo et al., 2013). El aporte de $\mathrm{N}$, proveniente de la FBN de las leguminosas probablemente, contribuyó al mantenimiento e incremento de dicho nutriente en el cultivo de girasol, que fue reflejado a través de los indicadores de producción del cultivo, en similitud a lo reportado por Boccolini et al. (2013).

Los mayores rendimientos del girasol fueron luego de V1 (2379 $\left.\mathrm{kg} \mathrm{ha}^{-1}\right)$, intermedios en $\mathrm{C}=\mathrm{BC}=\mathrm{V}_{2}=\mathrm{T}=\mathrm{A}+\mathrm{V}_{1}$ (promedio $\left.2023 \mathrm{~kg} \mathrm{ha}^{-1}\right)$ y los inferiores luego de A $\left(1561 \mathrm{~kg} \mathrm{ha}^{-1}\right)(\mathrm{p}=0,0673)($ Figura 8$)$. Estos resultados fueron similares a los ya obtenidos por Sá Pereira (2013) trabajando con Vicia villosa como antecesor de maíz en la región. Los rendimientos de girasol obtenidos con centeno como antecesor lo sugieren como una alternativa viable de contribución diferencial a otras gramíneas como la avena. Posiblemente, la precocidad de la variedad de centeno "Don Ewald" generó que la descomposición de su residuo se adelantara al momento de máximos requerimientos nutricionales del cultivo de renta. Los lixiviados de los residuos de CC por acción de la lluvia (aleloquímicos) contienen substancias orgánicas e inorgánicas que

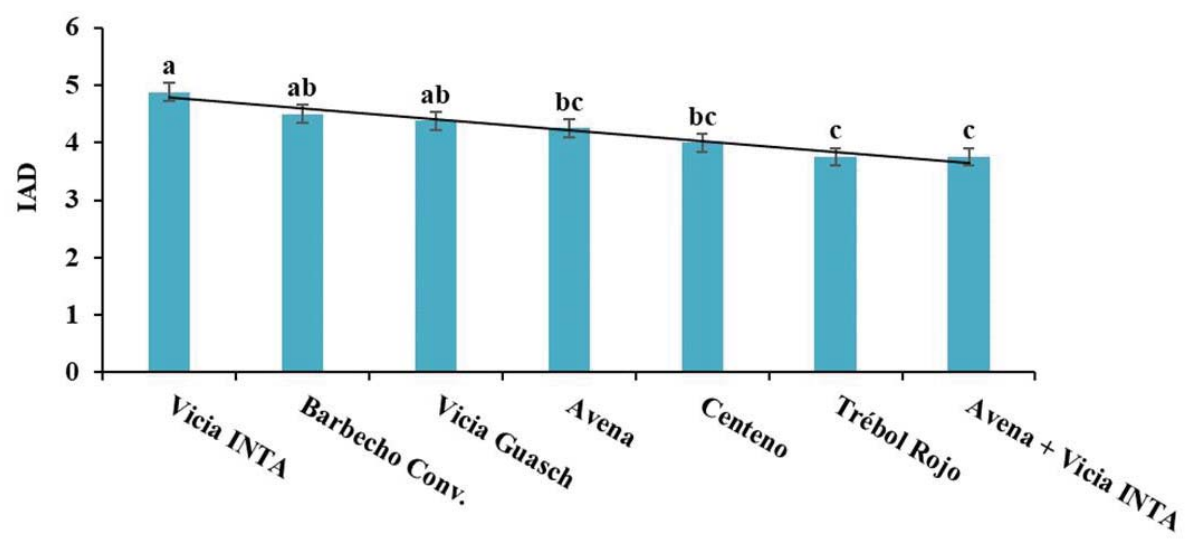

Antecesores Cultivos de cobertura

Figura 7: Índice de aspecto/desarrollo en prefloración del girasol con diferentes CC antecesor en escala desde $1=$ malo, $2=$ regular, $3=$ bueno, $4=$ muy bueno a $5=$ excelente. Letras distintas indican diferencias mínimas significativas $(\alpha \leq 0,05)$ según test de Fisher. Las lineas verticales en cada columna indican el error estándar de la media.

Figure 7: Pre-flowering aspect / development index of the sunflower crop with different ancestor CC in scale from $1=$ bad, $2=$ fair, $3=$ good, $4=$ very good $5=$ excellent. Different letters indicate minimal significant differences $(\alpha \leq 0.05)$ according to Fisher's test. Vertical line above each column indicates the standard error of the mean. 
pueden ser tanto tóxicas como inocuas o estimulantes (Almeida, 1988). En el centeno se destaca su capacidad de absorber nitratos residuales, aportar carbono e incrementar la cobertura del suelo. Ensayos realizados en diferentes regiones agrícolas de EE. UU., reportan que el centeno acumula entre 3500 y $5000 \mathrm{~kg} \mathrm{ha}^{-1}$ (25 a $75 \mathrm{~kg} \mathrm{~h}^{\mathrm{a}-1}$ ) (Smith et al., 1987). En trabajos sobre calidad de CC Sá Pereira et al. (2017 y 2017b), demostraron que, en el caso de avena, como antecesor de maíz, el aporte de material carbonado a los carbohidratos totales (CHt) del suelo, se demora en el tiempo y probablemente esta contribución de $\mathrm{CHt}$ al suelo en centeno se haya dado con anterioridad al período crítico. Por otro lado, existen evidencia de una mayor eficiencia de uso del agua del suelo luego del CC centeno como antecesor de maíz (González et al., 2017). Es destacable que los resultados obtenidos con $\mathrm{V}_{1} \mathrm{C}$ BC $\mathrm{y}_{2}$, bajo las condiciones de este sitio de ensayo, permitieron obtener rendimientos mayores $(p=0,06)$ (Figura 8). Estos supuestos deben ser confirmados en futuras investigaciones donde se trabaje con indicadores de calidad de CC centeno.

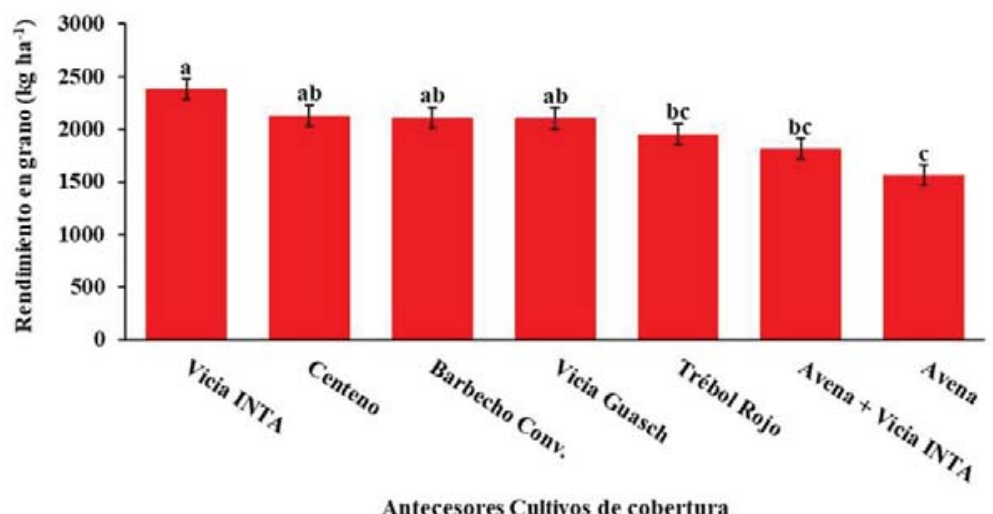

Figura 8: Producción de girasol (kg ha-1) con diferente CC antecesor. Letras distintas indican deferencias minimas significativas (Fisher, $\alpha \leq 0,10$ ). Las lineas verticales en cada columna indican error estándar de la media.

Figure 8: Sunflower production (kg ha-1) with different CC ancestors. Different letters indicate minimal significant differences (Fisher, $\alpha \leq 0.10$ ). Vertical lines above columns show standard error of the mean. 


\section{CONCLUSIONES}

Para las condiciones planteadas, en este ensayo con una secuencia de trigo/girasol continuo bajo SD sobre un Argiudol típico de Coronel Suárez, la inclusión de CC de vicia y centeno aumentaron la producción de materia seca y los rendimientos en grano de girasol, respecto a la situación de barbecho tradicional.

En cuanto a la producción de biomasa de los CC se encontraron diferencias importantes entre cultivos, las gramíneas fueron las que produjeron mayor cantidad respecto a las leguminosas.

Estos a su vez refleja mayor eficiencia en la utilización del agua de $\operatorname{los} \mathrm{CC}$, respecto al manejo del barbecho largo tradicional, comúnmente realizado por los productores del sudoeste bonaerense.

Los CC no redujeron significativamente el agua total del suelo al momento del secado respecto del testigo, donde no se encontraron diferencias importantes entre gramíneas y leguminosas.

Los resultados permiten concluir que es posible incluir $\mathrm{CC}$ en dicha rotación sin afectar la oferta hídrica del cultivo siguiente, en años con precipitaciones anuales dentro del promedio.

\section{AGRADECIMIENTOS}

Al Criadero "El Cencerro" por su colaboración en el montado y conducción de los ensayos y al Laboratorio de suelos del Departamento de Agronomía de la Universidad Nacional del Sur.

\section{BIBLIOGRAFIA}

Almeida FS. 1988. A alelopatia e as plantas. Londrina, IAPAR, $60 \mathrm{p}$.

Álvarez J, Scianca C. 2007. Cultivos de cobertura en Molisoles de la Región Pampeana. Aporte de carbono e influencia sobre las propiedades edáficas. EEA INTA General Villegas. Boletín para profesionales. Jornada profesional agrícola, 28 y 29 de septiembre del 2007.

Aulakh, MS, Doran JW, Walters DT, Mosier AR, Francis DD. 1991. Crop residue type and placement effects on denitrification and mineralization. Soil Sci. Soc. Am. J. 55:1020-1025.

Basanta MV, Giubergia JP, Lovera E, Alvarez C, Martellotto E, Curto E, Viglianco A. 2008. Manejo del barbecho invernal y su influencia en la disponibilidad hídrica para el cultivo estival en un Haplustol de la región central de Córdoba. XXI Congreso Argentino de la Ciencia del Suelo, San Luis. En CD.

Boccolini MF, Aimetta B, Cazorla C, Conde B. 2013. Efecto del residuo de vicia (Vicia sativa L.) sobre el potencial de nitrificación del suelo. En: Álvarez C, Quiroga A, Santos D, Bodrero M, editores. Contribuciones de los cultivos de cobertura a la sostenibilidad de los sistemas de producción. Anguil - La Pampa. Ediciones INTA. p 83-87.

Di Rienzo JA, Casanoves F, Balzarini MG, González L, Tablada M., Robledo CW. 2011. InfoStat, versión 2011, Grupo InfoStat, FCA, Universidad Nacional de Córdoba, Argentina.

González A, Eiza MJ, Carfagno P. 2017. Eficiencia del uso de agua y de barbechos en rotaciones con cultivos de cobertura. Rev. Fac. Agronomía y Cs. Agroalim. UM. VIII:95-111.

López MV, Arrúe JL. 1997. Growthyield and water use efficiency of winter barley in response to conservation tillage in a semi-arid region of Spain. Soil and Tillage Research. Vol. 44(1-2). Pág. 35-54. 
Mary B, Recous S, Darwis D, Robin D. 1996. Sá Pereira E de, Galantini JA, Quiroga AR. 2017.

Interactions between decompositions of plant residues and nitrogen cycling in soil. Plant and Soil. 18:171-82.

Mulvaney RL. 1996. Nitrogen-Inorganic forms. In: Methods of soil analysis. Part 3 Chemical Methods (D.L Sparks et al., ed) SSSA Book Ser. 5. Soil Sci. Soc. Am, Madison, 1123-1184.

Ranells NN, Wagger MG. 1996. Nitrogen release grass and legume cover crop monocultures and bicultures. Agron. J. 88:777-782.

RIAN Red de Información Agropecuaria Nacional. 2018. Informe INTA de cultivos de gruesa 2016-17 en el partido de Coronel Suárez. AER INTA Coronel Suárez.

Rillo S, Álvarez C, Bagnato R, Noellemeyer E. 2013. Cultivos de cobertura: gramíneas y leguminosas en el centro oeste de la provincia de Buenos Aires. En: Álvarez C, Quiroga A, Santos $\mathrm{D}$, Bodrero M, editores. Contribuciones de los cultivos de cobertura a la sostenibilidad de los sistemas de producción. Anguil - La Pampa. Ediciones INTA. p 58-68.

Smith, OB, Van Houtert MFJ, 1987. Valor forrajero de Gliricidia sepium. Reseña. Revista Mundial de Zootecnia, 47, 57-68.

Sá Pereira E de. 2013. Los cultivos de cobertura y la productividad del maíz en siembra directa: dinámica del nitrógeno, agua y fracciones orgánicas del suelo. Tesis Doctoral. Bahía Blanca. Universidad Nacional del Sur.

Sá Pereira E de, Galantini JA, Queiroga AR, Landriscini MR. 2014. Efecto de los cultivos de cobertura otoño invernales sobre el rendimiento y acumulación de $\mathrm{N}$ en maíz en el sudoeste bonaerense. Ciencia del suelo. 32(2):219-231.

Sá Pereira E. de, Álvarez C, Vechi G, Ibarra C, Pedelaborde JM. 2016. Cultivos de cobertura y barbechos de larga duración en sistemas agrícolas del sudoeste bonaerense. XXV Congreso Nacional de la Ciencia del Suelo. Río Cuarto. 27/06 al 01/07 de 2016. Actas.
Calidad de cultivos de cobertura en sistemas de siembra directa del sudoeste bonaerense. Ciencia del suelo. 35(2): 337-350.

Sá Pereira E de, Galantini JA, Duval ME. 2017a: Use of a three-compartment model to evaluate the dynamics of cover crop residues, Archives of Agronomy and Soil Science, DOI: 10.1080/03650340.2017.1296137. To link to this article: http://dx.doi.org/10.1080/0365034 0.2017.1296137. Use of a three-compartment model to evaluate the dynamics of cover crop residues. Archives of Agronomy and Soil Science. Sá Pereira E. de, Galantini JA, Duval E. 2017b. Decomposition from legume and non-legume crop residues: Effects on soil organic carbon fractions under controlled conditions. SJSS. SPANISH JOURNAL OF SOIL SCIENCE YEAR 2017 VOLUME 7 ISSUE:2

https://sjss.universia.net/article/view/2529/descomposicionrestos-cultivos-leguminosas-leguminosas-efectos-fracciones-carbono-organico-suelo-condicionescontroladas

SMN, 2018. Servicio meteorológico nacional. Aerostación Coronel Suárez https://www. smn.gob.ar/

Soil Survey Staff. 2014. Keys to soil taxonomy. USDA, Natura Resources Conservation Service. Washington, DC. EEUU. 372 p.

Thorup-Kristensen KJ, Magid J, Jensen LS. 2003. Catch crops and green manures as biological tools in nitrogen management in temperate zones. Advances in Agronomy 79: 227-302.

Trinsoutrot I, Recous S; Bentz B, Linères D, Chèneby D, Nicolardot B. 2000. Biochemical quality of crop residues and carbon and nitrogen mineralization kinetics under nonlimiting nitrogen conditions. Soil Sci. Soc. Am. J. 64:918-926.

Vanzolini J, Galantini JA, Agamenoni R, Martinez J, Reinoso O. 2010. Efecto del momento de secado de un cultivo de cobertura de Vicia villosa Roth. Sobre su acumulación de biomasa y su contenido de N. XXII Congreso Argentino de la Ciencia del Suelo - Rosario - (en CD). 